\title{
Diagnostic model for identification of myocardial infarction from electrocardiography signals
}

\author{
Aykut DİKER ${ }^{a}$, , Zafer CÖMERT ${ }^{b}$, Engin AVCIc \\ a Bitlis Eren University, Department of Informatics, TR-13000, Bitlis Turkey \\ b Bitlis Eren University, Department of Computer Engineering, TR-13000, Bitlis Turkey \\ c Firat University, Department of Software Engineering, TR-23000, Elazı̆̆, Turkey
}

\section{ART I C L E I N F O}

Article history:

Received 18 October 2017

Received in revised form 04 December 2017

Accepted 06 December 2017

\section{Keywords:}

Biomedical Signal Processing

Decision Support System

Machine Learning

Classification

Electrocardiography

\begin{abstract}
AB S T RACT
Electrocardiography (ECG) is a useful test used commonly to observe the electrical activity of a heart. Recently, a growing relationship has been observed between diagnosis of any heart disease and using of machine learning techniques. In this scope, a diagnostic application model designed based on a combination of Recursive Feature Eliminator (RFE) and two different machine learning algorithms called $k$-nearest neighbors $(k-\mathrm{NN})$ and artificial neural network (ANN) is proposed for classification of ECG signals in this study. The experiments performed on an open-access ECG database. Firstly, the signals were passed a pre-processing step. Then, several diagnostic features from morphological and statistical domains were extracted from ECG signals. In the last stage of the analysis, RFE algorithm covering 10fold cross-validation and the mentioned machine learning techniques were employed to separate Myocardial Infarction (MI) samples from normal. The promising results as accuracy of $80.60 \%$, sensitivity of $86.58 \%$ and specificity of $64.71 \%$ were achieved. The validation of the contribution was checked by comparing the performances of both $k-\mathrm{NN}$ and $\mathrm{ANN}$ to related works. Consequently, the proposed diagnostic model ensured an automatic and robust ECG signal classification model.
\end{abstract}

\section{Introduction}

Electrocardiogram (ECG) is a biomedical signal that provides information about the electrical activity of a heart (Ojha and Subashini 2014; Rahman and Nasor 2011; Sakarya and Arıca 2012). ECG signal consists of some critical diagnosis segments and wave types which are $\mathrm{P}$ wave, QRS complex and T wave as shown in Figure 1. During atrial depolarization, $\mathrm{P}$ wave occurs due to the compression of the blood from the atrium to the ventricle. QRS complex that is a combination of $Q, R$ and $S$ waves arises when ventricular depolarization happens. The duration of QRS complex is between $80 \mathrm{~ms}$ and $120 \mathrm{~ms}$ (longer than the duration of $\mathrm{P}$ wave). $\mathrm{T}$ wave following QRS complex represents ventricular repolarization (Chung-Ching Peng 2011). Analysis of ECG signal is an effective way to detect and treat different types of heart diseases. Cardiologists not only recognize the heart diseases using patient-specific waveforms in ECG signal but also they detect specific waveforms in ECG signal of a patient and ensure the basis for diagnosis.

\footnotetext{
* corresponding author: Aykut DIKER

Tel.: +90-541-907-2334

E-mail address: adiker@beu.edu.tr
} 
Furthermore, an ECG signal also includes several standard intervals that are the useful diagnostic parameters, such as QT, ST, and PR (Diker, Avcl, and Gedikpınar 2017).

Computer-based applications in biomedical signal processing are based on several fundamental steps such as preprocessing, feature transform covering extraction and selection of features, and classification (Cömert and Kocamaz 2016). When looking over previously reported studies, it can be seen that there are many feature extraction methods is used so as to describe ECG signals. Some of which are Wavelet transform (Khorrami and Moavenian 2010), Discrete Cosine Transform (DCT) (Chen et al. 2017), Wigner-Ville Distribution (Dash and Rao 2016). On the other hand, many researchers have used numerous techniques to reduce the dimension of feature space and to determine the most relevant features (Yang and Zhidong 2017). To this end, mutual information (MILCA, mRMR, NMIFS) (Valenzuela et al. 2013), genetic algorithms (GAs) (Silva Teodoro, Peres, and Lima 2017), multisession feature selection (MSFS) (Komeili et al. 2017), and dynamic programming (DP) algorithm (Acir 2005) have been employed. In addition, it is clear that many popular classification methods (Cömert and Kocamaz 2017a), such as fractal analysis (Lai and Chan 1998), chaotic modeling (Owis et al. 2002), bispectral coherence analysis (Khadra, Al-Fahoum, and Binajjaj 2005), and radial basis networks (Maglaveras et al. 1998) have been utilized successfully.

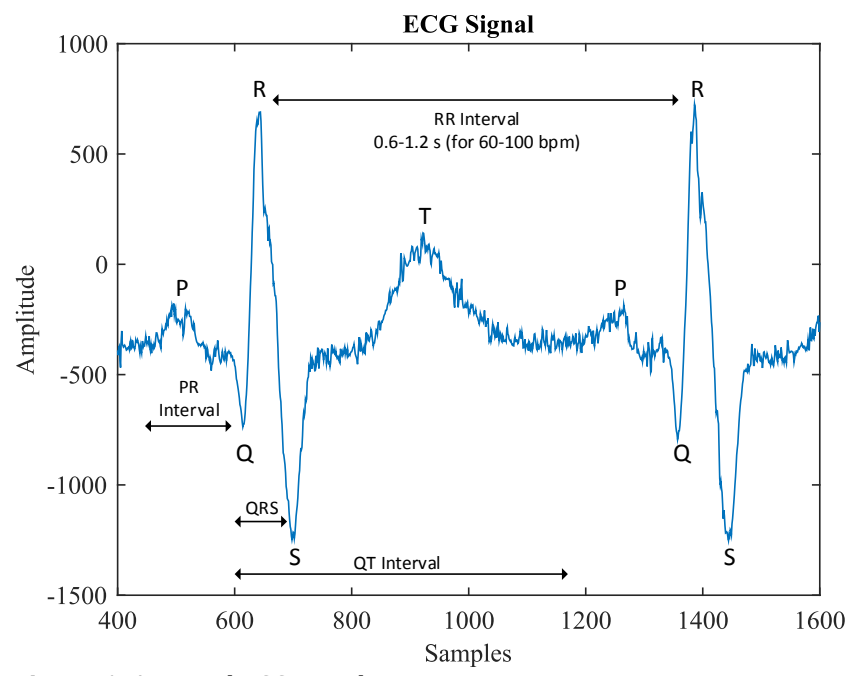

Figure 1. A typical ECG signal

In this study, we obtained several features from morphological and statistical domains to describe ECG signals after the preprocessing stage. In addition, in the next stage, we employed Recursive Feature Eliminator (RFE) algorithm to determine the most relevant features. In this stage of the analysis, $k$-nearest neighbors ( $k-\mathrm{NN}$ ) and artificial neural network (ANN) were employed as the models individually to classify ECG signals as normal and abnormal in 10-fold cross validation procedure. The primary objective of this contribution is to ensure a diagnostic model for identifying an abnormal heart disease known as Myocardial Infarction (MI).

The rest of the paper is organized as follows: the material and methods are summarized in Section 2. The experimental setups and results are presented in Section 3. A comparison between the proposed model and related works are given in Section 4. The concluding remarks are presented in Section 5.

\section{Material and Methods}

\subsection{Data collection}

In this study, a publicly available database called PhysikalischTechnische Bundesanstalt Diagnostic ECG Database (PTBDB) was used. The database was recorded with the help of the healthy volunteers and patients with different cardiac diseases in Benjamin Franklin University, Department of Cardiology in Berlin, Germany. The records in the database were collected from 290 subjects (each represented by 1 to 5 recordings) with a wide range of ages and diagnoses. In addition, the dataset consists of 549 recordings. Each of these recordings contains 15 simultaneously recorded signals: the conventional 12 leads (I, II, III, AVR, AVL, AVF, V1, V2, V3, V4, V5, V6) and the 3 Frank leads $\left(V_{x}, V_{y}, V_{z}\right)$. The recordings were digitized with 1000 samples per second per channel with 16-bit resolution at \pm $16,384 \mathrm{mV}$. Approximately $20 \%$ of subjects are healthy controls. The duration of each record is typically 2 minutes. Exceptionally, there is also a small number of shorter recordings, however, none of the records last less than 30 seconds. Diagnostic classification of recordings includes healthy and various disease categories such as MI, cardiomyopathy/heart failure, branch block, rhythm disturbance, myocardial and hypertrophy (Chen et al. 2013; Christov et al. 2006; M. Vozda, T. Peterek 2014). We performed experimental study only on normal and MI samples. Thus, 148 of MI and 52 of normal samples were selected from the database, respectively. In addition, all measurements were performed on only lead II in this study.

\subsection{Preprocessing}

The preprocessing is implemented as a standard analysis stage in many biomedical signal processing applications to achieve reliable and without noisy signals (Cömert and Kocamaz 2017b). ECG signals include some kinds of disturbance such as baseline wandering, electromyography noise, and power line interference. The low and derivative filters were used to resolve baseline wandering, In addition, $60 \mathrm{~Hz}$ noise and harmonic of the network was suppressed at this stage. After all operations, the noises remaining on the ECG signal were fixed using the Savitzky-Golay filter. 


\subsection{Feature Extraction}

After the preprocessing procedures, it is necessary to extract the features of ECG signal in order to use them in the classification stage.

Feature extraction is one of the most important stages in pattern recognition and classification tasks. In this study, eleven features in total were extracted from morphological and statistical domains.

The whole features are summarized in Table 1. In the feature extraction stage of ECG signal, R peaks were detected first. For this purpose, Pan-Tompkins algorithm, which is a good QRS complex peak detector, was used. Then, other critical points, such as PR interval, ST interval, QT interval (Cömert and Kocamaz 2015), and $P$ wave amplitude were detected by using Discrete Wavelet Transform (DWT). In addition, the statistical features which are Maximum (MAX), Minimum (MIN), Mean, Kurtosis, Skewness, Variance (VAR) were extracted from ECG signals.

Table 1. Features used in this study

\section{Morphological features}

(1) QRS complex amplitude (mV), (2) PR interval (ms), (3) ST interval (ms), (4) QT interval (ms), (5) P wave amplitude (mV)

Statistical features
(6) Maximum (MAX), (7) Minimum (MIN), (8) Mean, (9) Kurtosis,
(10) Skewness, (11) Variance (VAR)

\subsection{Feature Selection}

Feature selection is an important step for almost all machine learning algorithm. Using of smaller feature sets ensure simpler and more effective models which can produce better prediction performance (Webb 2003). At the end of the feature extraction stage, we obtained six features in total. RFE algorithm was employed to find a more informative subset used to apply as an input to the classifiers (Guyon et al. 2002). The steps followed by RFE algorithm are described as follows:

\begin{tabular}{lc}
\hline \multicolumn{2}{l}{ Algorithm 1: RFE algorithm } \\
\hline 01 & Train the model by using the whole features \\
02 & Measure the model performance \\
03 & Measure feature significance \\
04 & for each subset size $\boldsymbol{S}_{\boldsymbol{i}}, \boldsymbol{i}=\mathbf{1} \ldots \boldsymbol{S} \boldsymbol{d o}$ \\
05 & hold the $\boldsymbol{S}_{\boldsymbol{i}}$ most significant features \\
06 & train the model by using $\boldsymbol{S}_{\boldsymbol{i}}$ features \\
07 & measure model performance \\
08 & re-measure feature significance \\
09 & end for \\
10 & Measure the performance over $\boldsymbol{S}_{\boldsymbol{i}}$ \\
11 & Determine the proper number of features \\
12 & Employ the model based on the optimal $\boldsymbol{S}_{\boldsymbol{i}}$ \\
\hline
\end{tabular}

Each feature in the final set is evaluated considering its significance, and the features are ranked. Herein, $\boldsymbol{S}$ represents the ordered features $\left(\boldsymbol{S}_{1}>\boldsymbol{S}_{2} \ldots>\boldsymbol{S}\right)$.

\section{5. $k$-Nearest Neighbors}

\begin{tabular}{l} 
Algorithm 2: $k$-NN algorithm \\
\hline $01 \quad \begin{array}{l}\text { Adjust } k \text { as a nearest neighbors parameter } \\
02 \\
\text { Measure the distance between input-instance and all training } \\
\text { instances. }\end{array}$ \\
$03 \quad \begin{array}{l}\text { Order the distance and discover nearest neighbors } \\
\text { considering }{ }^{\text {th }} \text { minimum distance }\end{array}$ \\
$\begin{array}{l}\text { Collect the category of the nearest neighbors } \\
\text { Evaluate the simple majority of the category of nearest } \\
\text { neighbors for the input-instance }\end{array}$
\end{tabular}

$\boldsymbol{k}$-NN is a non-parametric supervised classifier. Its primary objective is to predict an unknown instance by using its $\boldsymbol{k}$ nearest neighbors that are stored in a training set (Aha, Kibler, and Albert 1991). For this particular purpose, the algorithm utilizes the several distance functions such as Euclidean and follows the procedures as described before in Algorithm 2.

\subsection{Artificial Neural Network}

ANN is a virtual computational model of the human nervous system that consists of multilayers structure. In general, an ANN model consists of an input layer feeding the classifier, one or more hidden layer(s) used to catch nonlinearity in the studied data space, and an output layer producing a numeric value for an estimation or class information (Hagan et al. 2014). ANN tries to map inputs to outputs with a learning process updating the weights and biases. This multilayer computational structure can be modeled as follows:

$$
h_{i}=\sigma\left(\sum_{j=1}^{N} V_{i j} x_{j}+T_{i}^{\text {hidden }}\right)
$$

where $\boldsymbol{\sigma}($.$) is used to express activation function, \boldsymbol{N}$ shows the number of neurons. Also $\boldsymbol{V}_{\boldsymbol{i} \boldsymbol{j}}$ symbolizes the weights, $\boldsymbol{x}_{\boldsymbol{j}}$ is inputs and $\boldsymbol{T}_{\boldsymbol{i}}^{\text {hidden }}$ is the threshold value for the hidden nodes.

\section{Experimental Results}

This section presents the classification results for control and case recordings. An experimental study was realized using MATLAB $^{\circledR}(2017 a)$ software.

\subsection{Performance Evaluation Criteria}

$k$-fold cross-validation, confusion matrix, receiver operating characteristic (ROC) curve are the basic evaluation tools to measure the performance of the models. 
$k$ - fold cross-validation ensures a better generalization performance by preventing the picking a particular part from the studied data. The data is divided into $k$ parts, and the evaluation process is repeated at $k$ times in this method. In each round, the certain parts of data are separated into the training and test sets, and the $k-1$ part of the data is used for training and the rest is used for testing. At the end of $k$ round, the average performance values are obtained. It should be noted that $k$-fold cross validation becomes an important tool especially when the amount of data to be used for analysis is small (Jonathan, Krzanowski, and McCarthy 2000).

Confusion matrix, also often called error matrix, contains four prognostic indexes which are True Positive (TP), True Negative (TN), False Positive (FP), and False Negative (FN). TP and TN show the number of samples predicted correctly for positive and negative samples, respectively (Powers, David 2011). Similarly, FP and FN show the number of samples predicted incorrectly for positive and negative samples, respectively. Confusion matrix also ensures calculating the several performance metrics, some of which are given as follows:

$$
A c c=\frac{T P+T N}{T P+F P+F N+T N}
$$

Accuracy $(A C c)$ corresponds the overall performance of the model.

$$
S e=\frac{T P}{T P+F N}
$$

Sensitivity $(\mathrm{Se}$ ) a significant metric to understand the model performance on identifying positive samples.

$$
S p=\frac{T N}{T N+F P}
$$

Specificity $(S p)$ indicates the ratio of a negative test result for the model.

$$
Q I=\sqrt{S e \cdot S p}
$$

Quality Index $(Q I)$ describing the geometric mean of $S e$ and $S p$ is another beneficial metric, especially when the number of samples among the classes is not equal.

It should be noted that an experiment may report biased results when the distribution of data among the classes is imbalanced. ROC curve expresses the classifier performance without considering class distribution or error costs. The area under this curve (AUC) is used as a performance metric in this study.

\subsection{Experimental Results}

The main aim of this study is to provide a support decision model or a diagnostic model for discriminating MI samples from normal. To this end, a total 200 recordings consisting of $152 \mathrm{MI}$ and 48 normal samples were selected from an openaccess PTB database. All of the signals were represented with 11 diagnostic features as described in Table 1. After the feature extraction stage, the statistical values of each feature are obtained as given in Table 2 for MI and normal samples, individually.

Table 2. Features used in this study

\begin{tabular}{lcc}
\hline Features & $\begin{array}{c}\text { MI } \\
(\mathbf{N = 1 4 8 )}\end{array}$ & $\begin{array}{c}\text { Normal } \\
(\mathbf{N}=\mathbf{5 2})\end{array}$ \\
\hline QRS amplitude & $1.04 \pm 0.44$ & $1.45 \pm 0.23$ \\
PR interval & $-0.68 \pm 0.41$ & $-0.50 \pm 0.25$ \\
ST interval & $11.95 \pm 6.89$ & $15.28 \pm 6.57$ \\
QT interval & $1.11 \pm 2.09$ & $2.79 \pm 1.08$ \\
P amplitude & $0.06 \pm 0.03$ & $0.07 \pm 0.02$ \\
Max & $0.86 \pm 0.13$ & $0.95 \pm 0.05$ \\
Min & $1.66 \pm 0.51$ & $2.21 \pm 0.65$ \\
Mean & $1.44 \pm 0.50$ & $1.99 \pm 0.64$ \\
Kurtosis & $0.41 \pm 0.03$ & $0.41 \pm 0.03$ \\
Skewness & $0.43 \pm 0.04$ & $0.44 \pm 0.03$ \\
Variance & $1.52 \pm 0.51$ & $2.07 \pm 0.65$ \\
\hline
\end{tabular}

In the first evaluation, all the features in the feature set were used to train ANN and $k$-NN networks. A special type of neural network called as pattern recognition network was used in the experiment. In order to configure the network, the network topology was adjusted to 11 input nodes, one hidden layer with 12 nodes and 2 output nodes. The entire of data was divided into two sets as training and test in 10 -fold cross-validation procedure. In this scope, $90 \%$ of data was used for training while the rest of data was used for testing in each round, as mentioned before. By the way, the validation set was not used in the evaluation process. Also, initial momentum $(\mathrm{mu}), \mathrm{mu}$ decrease factor and $m u$ increase factor, and maximum $m u$ were set as $0.001,0.1,10$, and $1 \mathrm{e} 10$, respectively for ensuring a fast training. Consequently, the results of ANN with the different number of repetitions (Rp) are given in Table 3.

Table 3. The results of ANN with different number of repetitions

\begin{tabular}{ccccc}
\hline Rp & Acc (\%) & Se (\%) & Sp (\%) & QI (\%) \\
\hline 1 & $77.78 \pm 10.9$ & $87.09 \pm 13.1$ & $56.47 \pm 32.6$ & $63.97 \pm 27.7$ \\
10 & $80.10 \pm 8.52$ & $86.29 \pm 8.89$ & $62.40 \pm 23.1$ & $71.22 \pm 17.7$ \\
20 & $80.20 \pm 8.27$ & $86.56 \pm 8.71$ & $60.95 \pm 24.9$ & $69.39 \pm 20.2$ \\
30 & $80.43 \pm 8.80$ & $87.04 \pm 8.72$ & $62.48 \pm 21.4$ & $72.06 \pm 15.3$ \\
40 & $80.62 \pm 8.53$ & $86.49 \pm 9.07$ & $64.33 \pm 22.2$ & $72.76 \pm 15.9$ \\
50 & $80.26 \pm 8.60$ & $86.48 \pm 8.75$ & $63.35 \pm 23.7$ & $71.64 \pm 18.1$ \\
\hline
\end{tabular}


Mean \pm Standard deviation

Table 4. The aggregate confusion matrix of the ANN model with 50 repetitions

\begin{tabular}{cccc}
\hline TP & FP & FN & TN \\
\hline 6348 & 940 & 1032 & 1660 \\
\hline
\end{tabular}

It is clear that the models are capable of classifying case recordings (MI samples) but not control recordings (normal samples). The basic reason for this situation is imbalanced data distribution among the classes. As mentioned before, we have 152 MI samples and only 48 normal samples. Therefore, sensitivity values were obtained fairly high while specificity values were low. In addition, it should be noted that the reporting of only the accuracy metric will be misleading when imbalanced data is used for evaluating a model. This situation is demonstrated in Figure 2. As seen in Figure 2, the average values of accuracy were indicated at almost $80 \%$. In fact, the performance of the model is not that high. In such cases, using of F-measure or QI metrics is more suitable for evaluating the model. As a result, the model of ANN to which all features are applied as input has reached a value of $71.64 \%$ QI. The aggregate confusion matrix of the model with 50 repetition is given in Table 4.

As for $k$-NN, we evaluated $k$ value range in 1 to 10 , first. $k$ value was adjusted to 3 considering the experimental results. Then, we tested the $k$-NN network with different number of repetition. The obtained results are summarized in Table 5 , the aggregate confusion matrix of the $k$-NN model with 50 repetition is presented in Table 6 , and the results is shown in Figure 3, respectively.

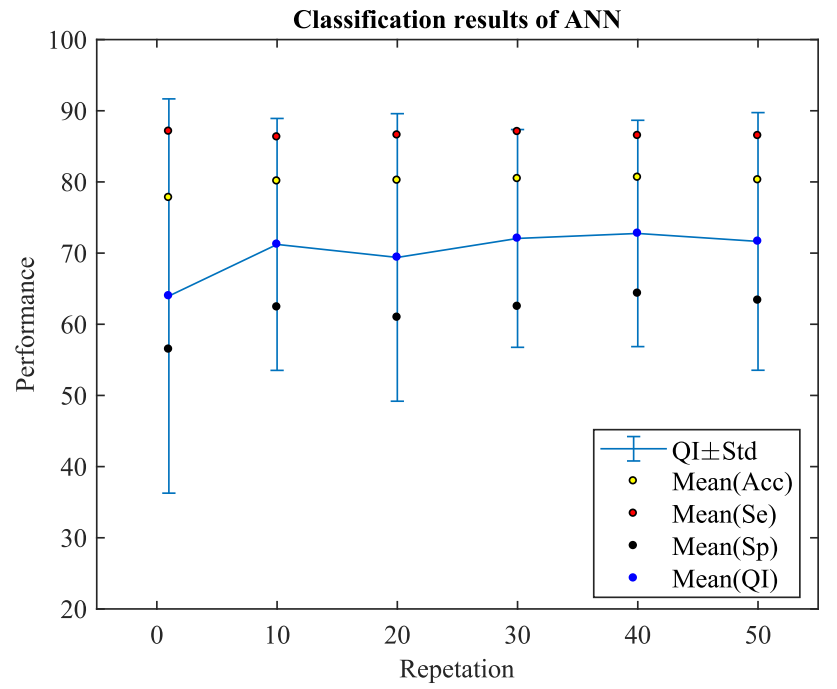

Figure 2. The results of ANN with different number of repetitions
When the classification results of ANN and $k$-NN are compared, it is clearly seen that ANN is superior to $k-\mathrm{NN}$.

In the last stage of the experiments, we evaluated the discriminatory capacity of the each feature by using RFE algorithm. The various feature combinations were investigated to increase the performances of the models.

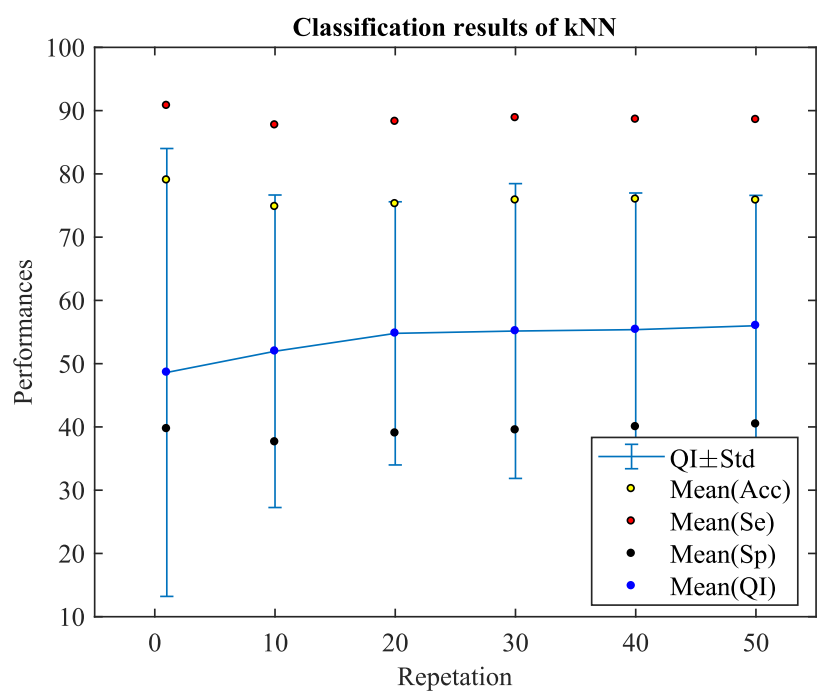

Figure 3. The results of $k$-NN with different number of repetitions

Table 5. The results of $\boldsymbol{k}$-NN with different number of repetitions

\begin{tabular}{ccccc}
\hline Rp & Acc (\%) & Se (\%) & Sp (\%) & QI (\%) \\
\hline 1 & $79.00 \pm 8.75$ & $90.78 \pm 11.4$ & $39.69 \pm 32.1$ & $48.61 \pm 35.4$ \\
10 & $74.80 \pm 9.31$ & $87.70 \pm 8.59$ & $37.60 \pm 24.4$ & $51.96 \pm 24.7$ \\
20 & $75.25 \pm 8.71$ & $88.26 \pm 8.27$ & $39.00 \pm 20.9$ & $54.79 \pm 20.8$ \\
30 & $75.83 \pm 9.76$ & $88.84 \pm 8.70$ & $39.49 \pm 23.9$ & $55.16 \pm 23.3$ \\
40 & $75.97 \pm 9.05$ & $88.60 \pm 8.55$ & $40.00 \pm 22.1$ & $55.38 \pm 21.6$ \\
50 & $75.82 \pm 8.51$ & $88.55 \pm 7.75$ & $40.43 \pm 22.4$ & $56.00 \pm 20.6$ \\
\hline
\end{tabular}

Mean \pm Standard deviation

Table 6. The aggregate confusion matrix of the $\boldsymbol{k}$-NN model with 50 repetition

\begin{tabular}{cccc}
\hline TP & FP & FN & TN \\
\hline 6548 & 1566 & 852 & 1034 \\
\hline
\end{tabular}

As a result, the eleven features were ranked considering their discriminative capacity by RFE algorithm as (1) Maximum, (2) QRS complex amplitude, (3) Minimum, (4) Mean, (5) Variance, (6) QT interval, (7) Kurtosis, (8) Skewness, (9) P wave amplitude, (10) ST interval and (11) PR interval.

Considering the discriminative capacity of the each feature, the dimension of feature set was increased gradually and the obtained results are reported in Table 7. The mean QI values 
corresponding to the number of used features for classifying are illustrated in Figure 4.

After the feature selection procedure, the feature set consisting of six features: Maximum, QRS complex amplitude, Minimum, Mean, Variance and QT interval was found as the most informative. The best classification performance that was reached using all features was surpassed by using only specified six features in the same feature set.

Table 7. RFE feature ranking performances

\begin{tabular}{ccccc}
\hline F\# & Acc (\%) & Se (\%) & Sp (\%) & QI (\%) \\
\hline 1 & $76.70 \pm 7.85$ & $82.53 \pm 9.77$ & $61.11 \pm 22.7$ & $68.71 \pm 15.6$ \\
2 & $74.94 \pm 9.59$ & $81.99 \pm 10.9$ & $57.72 \pm 22.9$ & $66.56 \pm 16.2$ \\
3 & $78.45 \pm 8.30$ & $86.59 \pm 8.24$ & $55.85 \pm 25.2$ & $66.72 \pm 19.7$ \\
4 & $80.41 \pm 8.41$ & $88.42 \pm 8.01$ & $61.07 \pm 23.4$ & $71.32 \pm 16.9$ \\
5 & $80.35 \pm 8.94$ & $88.26 \pm 9.08$ & $58.31 \pm 24.7$ & $68.68 \pm 20.7$ \\
6 & $80.60 \pm 8.56$ & $86.58 \pm 9.53$ & $64.71 \pm 22.9$ & $72.72 \pm 16.6$ \\
7 & $80.35 \pm 7.82$ & $85.69 \pm 8.56$ & $64.86 \pm 23.4$ & $72.23 \pm 18.1$ \\
8 & $79.15 \pm 9.01$ & $85.07 \pm 10.1$ & $63.81 \pm 21.2$ & $71.99 \pm 15.8$ \\
9 & $78.80 \pm 9.04$ & $84.56 \pm 10.7$ & $62.14 \pm 21.2$ & $70.54 \pm 15.8$ \\
10 & $79.20 \pm 7.90$ & $85.95 \pm 9.11$ & $60.17 \pm 23.6$ & $69.65 \pm 16.7$ \\
11 & $80.50 \pm 9.17$ & $87.05 \pm 8.49$ & $62.19 \pm 21.7$ & $72.07 \pm 15.4$ \\
\hline
\end{tabular}

Mean + standard deviation. F\#: the number of used features

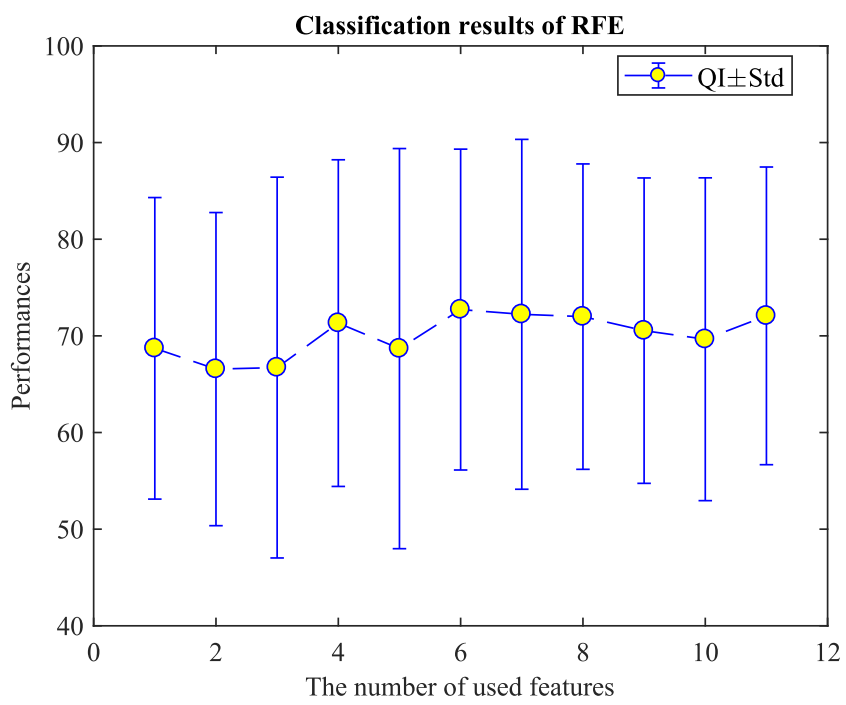

Figure 4. The QI performance results of RFE

In the end of a comprehensive experimental study, as mentioned before, we achieved six informative features. Finally, this subset was used to feed the pattern recognition network and the average AUC value was obtained as 0.81 for MI samples, shown in Figure 5.

\section{Discussion}

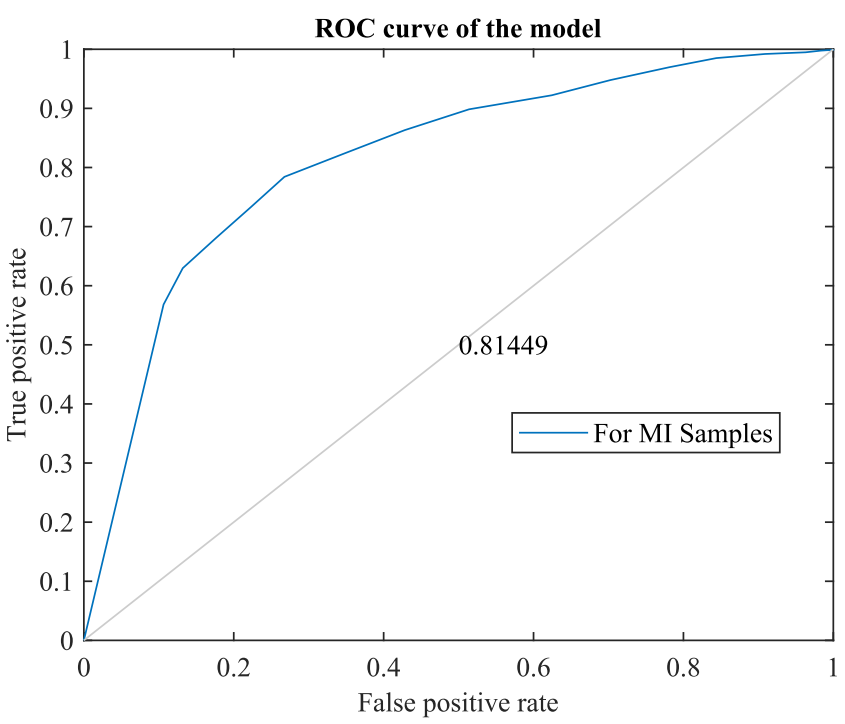

Figure 5. The ROC curve of the model with RFE

First of all, it should be noted that making a one-to-one comparison between the proposed model and related works is fairly difficult due to the existing of different features, methods, a variable number of recordings, etc. as indicated in Table 8 . According to the results in Table 8 , it is clear that the performance of the proposed clinical decision support system of ECG analysis should be improved.

(Chen et al. 2013) conducted a comparative study focusing on spectral energy features and based on several classifiers. As seen in Table 8 , they achieved quite promising results as sensitivity of $91.30 \%$ and specificity of $84.31 \%$.

(Noorianl, Dabanloo, and Parvaneh 2014) suggested a model used deep features considering three classes. A really high classification performance was obtained. The sensitivity and specificity values of the study were reported as $99.60 \%$ and $99.82 \%$, respectively.

(Kulkarni 2015) performed an experimental study with a small number of recordings. The morphological features were taken into account in the study, and ANN was employed as a classifier. Sensitivity was indicated as $89.91 \%$ and specificity was indicated as $94.38 \%$ in the study.

In this study, we considered only morphological and statistical features of ECG signals, and only a single channel was preferred for the analysis. As plainly seen in the related works, it may be possible to raise the performance of the model using different diagnostic features extracted from different domains such as nonlinear and time-frequency. Also, consideration of multiple channels may also be useful. 
Table 8. A comparison of the classification results for ECG analysis using different algorithms

\begin{tabular}{|c|c|c|c|c|c|c|}
\hline References & Database & Features & Classifier & \# Classes & Se (\%) & Sp (\%) \\
\hline (Chen et al. 2013) & $\begin{array}{l}\text { PTB } 104 \text { records, } \\
\text { SGHCD } 17 \text { records }\end{array}$ & $\begin{array}{l}\text { Spectral energy } \\
\text { features }\end{array}$ & LDA, QDA, SVM, $k$-NN & 2 & 91.30 & 84.31 \\
\hline (Noorianl et al. 2014) & PTB 549 records & Deep Features & $\begin{array}{l}\text { Multi-Scale Deep } \\
\text { Feature }\end{array}$ & 3 & 99.60 & 99.82 \\
\hline (Kulkarni 2015) & $\begin{array}{l}\text { MIT-BIH } 48 \\
\text { records }\end{array}$ & Morphological & ANN & 3 & 89.91 & 94.38 \\
\hline This paper & PTB 200 records & $\begin{array}{l}\text { Morphological and } \\
\text { statistical features }\end{array}$ & ANN, RFE, CV & 2 & 86.51 & 64.71 \\
\hline
\end{tabular}

\section{Conclusion}

The primary aim of this paper was to ensure a model which has capable of distinguishing MI samples from normal strongly. The results show that it is possible to achieve $80.60 \%$ for accuracy, $86.58 \%$ for specificity, $64.74 \%$ for sensitivity and $72.72 \%$ for the quality index by using the pattern recognition network. The results of this study show that it may be developed more effective models for identifying MI samples as well as normal samples. Nonetheless, the reported results are encouraging considering the discriminative capacity of the model on MI recordings. On the other hand, the study needs more evaluation using independent and larger datasets.

In the future work, it will be also meaningful to assess different diagnostic features extracted from different domains such as time-frequency, nonlinear, or DWT. Apart from diagnostic features, taking the performances of advanced machine learning techniques such as extreme learning machine and support vector machine will be notable.

Consequently, the proposed diagnostic model is robust, contributes to identifying a pathological heart disease called as MI, automatically and ensures new insights into the evaluation of machine learning algorithms for analyzing ECG signals.

\section{Acknowledgements}

The dataset used in this work is shared on an online research platform called www.ctganalysis.com. We would like to thank the researchers designed this research platform.

\section{References}

Acir, N., 2005. Classification of ECG Beats by Using a Fast Least Square Support Vector Machines with a Dynamic Programming Feature Selection Algorithm. Neural Computing and Applications 14(4):299309.

Aha, D. W., Dennis K., and Marc K. A., 1991. Instance-Based Learning Algorithms. Machine Learning 6(1):37-66.

Chen, S., Wei H., Zhi L., Jian L., and Xingjiao G., 2017. Heartbeat Classification Using Projected and Dynamic Features of ECG Signal. Biomedical Signal Processing and Control 31:165-73.
Chen, T., Evangelos B., Mazomenos, K. M., Srinandan D, and Mahesan N., 2013. Design of a Low-Power on-Body ECG Classifier for Remote Cardiovascular Monitoring Systems. IEEE Journal on Emerging and Selected Topics in Circuits and Systems 3(1):75-85.

Christov, I., et al., 2006. Dataset of Manually Measured QT Intervals in the Electrocardiogram. BioMedical Engineering Online 5 (Table 4):31.

Chung-Ching P., 2011. A Memory-Optimized Archticeture for ECG Signal Processing. Universty of Florida.

Cömert, Z., and Kocamaz A. F., 2015. Determination of QT Interval on Synthetic Electrocardiogram. 23rd Signal Processing and Communications Applications Conference (SIU).

Cömert, Z., and Kocamaz, A. F., 2017a. Comparison of Machine Learning Techniques for Fetal Heart Rate Classification. Acta Physica Polonica A 132(3):451-54.

Cömert, Z., and Kocamaz, A. F., 2017b. Using Wavelet Transform for Cardiotocography Signals Classification. 25th Signal Processing and Communications Applications Conference (SIU).

Cömert, Z., and Kocamaz. A.F., 2016. Evaluation of Fetal Distress Diagnosis during Delivery Stages Based on Linear and Nonlinear Features of Fetal Heart Rate for Neural Network Community. International Journal of Computer Applications 156(4):26-31.

Dash, S. K., and Sasibhusan R., 2016. Arrhythmia Detection Using Wigner-Ville Distribution Based Neural Network. Procedia Computer Science, vol. 85. Elsevier Masson SAS.

Diker, A., Avcı, E., and Gedikpınar M., 2017. Determination of R-Peaks in ECG Using Hilbert Transform and Pan-Tompkins Algorithms. 25th Signal Processing and Communications Applications Conference (SIU) (2017).

Guyon, I, Jason W., Stephen B., and Vladimir V., 2002. Gene Selection for Cancer Classification Using Support Vector Machines. Machine Learning 46(1):389-422.

Hagan, M. T., Howard, B. D., Mark, H., B., and Orlando, De J., 2014. Neural Network Design. Martin Hagan. 
Jonathan, P., Krzanowski, W. J., and McCarthy W. V., 2000. On the Use of Cross-Validation to Assess Performance in Multivariate Prediction. Statistics and Computing 10(3):209-29.

Khadra, L., Amjed S. Al-F., and Saed B., 2005. A Quantitative Analysis Approach for Cardiac Arrhythmia Classification Using Higher Order Spectral Techniques. IEEE Transactions on Biomedical Engineering 52(11):1840-45.

Khorrami, H., and Majid, M., 2010. A Comparative Study of DWT, CWT and DCT Transformations in ECG Arrhythmias Classification. Expert Systems with Applications 37(8):5751-57.

Komeili, M., Louis, W., Armanfard, N., and Hatzinakos, D., 2017. Feature Selection for Nonstationary Data: Application to Human Recognition Using Medical Biometrics. IEEE Transactions on Cybernetics PP(99):114.

Kulkarni, S. P., 2015. DWT and ANN Based Heart Arrhythmia Disease Diagnosis from MIT-BIH ECG Signal Data. International Journal on Recent and Innovation Trends in Computing and Communication, Vol. 3, No. 1, pp. 276-279.

Lai, K. T., and Chan, K. L., 1998. Real-Time Classification of Electrocardiogram Based on Fractal and Correlation Analyses. Proceedings of the 20th Annual International Conference of the IEEE Engineering in Medicine and Biology Society. Vol.20 Biomedical Engineering Towards the Year 2000 and Beyond (Cat. No.98CH36286) 20(1):119-22.

Maglaveras, N., Stamkopoulos T., Diamantaras, K., Pappas C., and Strintzis, M., 1998. ECG Pattern Recognition and Classification Using Non-Linear Transformations and Neural Networks: A Review. Int. J Med. Inform. 52(1-3):191-208.

Noorianl, A., Nader D., and Saman, P., 2014. Wavelet Based Method for Localization of Myocardial Infarction Using the Electrocardiogram.
Ojha, Durgesh Kumar and Monica Subashini. 2014. Analysis of Electrocardiograph ( ECG) Signal for the Detection of Abnormalities Using MATLAB. International Journal of Medical, Health, Biomedical and Pharmaceutical Engineering 8(2):114-17.

Owis, M. I., Abou-Zied, A. H., Abou, B., Youssef M., and Kadah, Y. M., 2002. Study of Features Based on Nonlinear Dynamical Modeling in ECG Arrhythmia Detection and Classification. IEEE Transactions on Biomedical Engineering 49(7):733-36.

Powers, D., 2011. Evaluation: From Precision, Recall and F-Measure to Roc, Informedness, Markedness \& Correlation. Journal of Machine Learning Technologies 2(1):37-63.

Rahman, M., and Nasor M., 2011. An Algorithm for Detection of Arrhythmia. 1st Middle East Conference on Biomedical Engineering, vol. 2.

Sakarya, C., and Arıca, S., 2012. QRS Detection with Wavelet Transform Using A Custom Wavelet. 396-400.

Silva T., Felipe G., Sarajane M. P., and Clodoaldo A. M. L., 2017. Feature Selection for Biometric Recognition Based on Electrocardiogram Signals. International Joint Conference on Neural Networks (IJCNN), IEEE.

Valenzuela, O., et al. 2013. Intelligent Systems to Autonomously Classify Several Arrhythmia Using Information from ECG. International Conference Social Computing (SocialCom).

Vozda, M., Peterek, T., Cerny, M., 2014. Novel Method for Deriving Vectorcardiographic Leads Based on Artificial Neural Networks. Webb, A. R., 2003. Statistical Pattern Recognition. John Wiley \& Sons.

Yang, Z. and Z. Zhidong. 2017. "Fetal State Assessment Based on Cardiotocography Parameters Using PCA and AdaBoost." Fetal State Assessment Based on Cardiotocography Parameters Using PCA and AdaBoost (October). 